\title{
Afamin serum concentrations are associated with insulin resistance and metabolic syndrome in polycystic ovary syndrome
}

\author{
Beata Seeber ${ }^{1}$, Elisabeth Morandell ${ }^{1}$, Fabian Lunger ${ }^{1}$, Ludwig Wildt ${ }^{1}$ and Hans Dieplinger ${ }^{2,3^{*}}$
}

\begin{abstract}
Background: High plasma concentrations of the vitamin E-binding protein afamin have been previously shown to be associated with insulin resistance and metabolic syndrome. We set out to determine whether the concentration of afamin in the serum of women with polycystic ovarian syndrome (PCOS) is elevated in relation to the presence and severity of insulin resistance (IR).
\end{abstract}

Methods: This cross-sectional study looked at 53 patients with PCOS and 49 non-PCOS patients. IR was diagnosed as a HOMA Index $>2.4$ and confirmed with a three-hour glucose tolerance test. Serum concentrations of afamin were determined using enzyme-linked immunosorbent assay (ELISA). Clinical characteristics, hormone and metabolic parameters were correlated to afamin concentrations.

Results: Serum concentrations of afamin did not differ between women with PCOS and controls. When separated according to the presence of IR a significant difference in median afamin levels was seen between PCOS with IR and PCOS without IR $(73.06+/-27.36 \mathrm{mg} / \mathrm{L}$ and $64.25+/-17.41 \mathrm{mg} / \mathrm{L}, \mathrm{P}=0.033)$. No difference in afamin levels was detected when comparing the few controls with IR and the controls without IR $(76.20+/-27.96 \mathrm{mg} / \mathrm{L}$ and $60.44+/-21.03 \mathrm{mg} / \mathrm{L}$, $\mathrm{p}=0.235$ ). On univariate analyses, afamin serum concentrations significantly correlated with BMl, triglycerides, HOMA Index, and AUC-Insulin. On multivariate linear regression analysis, only triglyceride concentration was seen to be an independent predictor of afamin. Subjects with metabolic syndrome had higher median afamin concentrations than did those without metabolic syndrome $(77.43+/-28.60 \mathrm{mg} / \mathrm{L}$ and $65.08+/-18.03 \mathrm{mg} / \mathrm{L}, \mathrm{P}=0.010)$.

Conclusions: Elevated afamin concentrations are associated with the presence of metabolic syndrome in young women and may potentially serve as an independent predictor for the development of metabolic syndrome in at-risk women, especially those with IR.

Keywords: Afamin, Polycystic ovary syndrome, Insulin resistance, Metabolic syndrome

\section{Background}

Polycystic ovary syndrome (PCOS) was first described in 1935 by Stein and Leventhal in patients with amenorrhoea, hirsutism, obesity and histological evidence of polycystic ovaries [1]. PCOS is a heterogeneous syndrome of unclear etiology with a prevalence of up to $20 \%$, making it the most frequent endocrine disorder in women of reproductive age $[2,3]$. There exist, however,

\footnotetext{
* Correspondence: hans.dieplinger@i-med.ac.at

${ }^{2}$ Department of Medical Genetics, Division of Genetic Epidemiology,

Molecular and Clinical Pharmacology, Innsbruck Medical University,

Schöpfstrasse 41, A-6020 Innsbruck, Austria

${ }^{3}$ Vitateq Biotechnology GmbH, Innsbruck, Austria

Full list of author information is available at the end of the article
}

no universal diagnostic criteria for PCOS [4], although most clinicians and researchers have accepted the definition published after the consensus conference in Rotterdam in 2003 [5]. Women are diagnosed with PCOS if they have at least two of the following criteria: a) oligo- or amenorrhoea, b) hyperandrogenemia or clinical signs of hyperandrogenism, c) sonographically diagnosed polycystic ovaries, after exclusion of other related disorders.

The pathogenesis of PCOS is a complex mixture of genetic and environmental origins [4]. Insulin resistance (IR) and compensatory hyperinsulinemia are observed in as many as $50 \%-60 \%$ of PCOS women [6]. Moreover, women with PCOS exhibit a four-fold higher incidence of metabolic syndrome. The prevalence of metabolic 
syndrome in PCOS is reported to be $8 \%-50 \%$, depending on study design and population [7-9].

Metabolic syndrome has been increasingly diagnosed in the general population in recent decades. It is defined as a combination of risk factors for type 2 diabetes mellitus (T2DM) and cardiovascular disease (CVD), including dyslipidemia, hyperglycemia, abdominal obesity, and hypertension [10], three or more of which are required for the diagnosis [11]. According to data from the third National Health and Nutrition Examination Survey (NHANES III, 1988-1994), the overall prevalence of patients with manifest metabolic syndrome was $22 \%$, increasing with age and depending on sex and ethnic origin [12]. If untreated, metabolic syndrome likely results in the early development of life-threatening sequelae such as T2DM, CVD and stroke [13-17]. It is not yet clear, however, to what degree these risk profiles are associated with cardiovascular outcomes in women with PCOS [18].

Very recently, the human plasma vitamin E-binding protein afamin was reported to be highly significantly associated with criteria for metabolic syndrome in three independent human general populations [19]. In that work, data from a prospective study design as well as corresponding data from afamin-transgenic mice suggest a role of afamin in the development of metabolic syndrome.

Afamin was discovered in 1994 as the fourth member of the human albumin gene family, which includes human serum albumin, alpha-fetoprotein and vitamin D-binding protein [20]. Afamin is a human plasma glycoprotein of 87 $\mathrm{kD}$ with $15 \%$ carbohydrate content and 55\% amino acid sequence similarity to albumin. Circulating plasma afamin is primarily of hepatic origin [20]. Abundant concentrations of afamin have been described in plasma and other body fluids such as follicular, cerebrospinal and seminal fluid [21].

We previously demonstrated multiple vitamin Ebinding sites of human afamin using a radio-ligand assay [22]. Afamin concentrations were found to correlate with those of vitamin $E$ in follicular and cerebrospinal fluids, but not in plasma [21]. This may indicate a dissociation of afamin from its vitamin $\mathrm{E}$ carrier function in plasma, unlike its physiological carrier function in follicular and cerebrospinal fluids.

Prompted by these observations, we measured afamin concentrations in patients with and without PCOS and investigated the association between afamin, IR and metabolic syndrome as a first step in establishing afamin as a marker for the development of metabolic syndrome in PCOS women.

\section{Methods \\ Subjects}

Eligible subjects for this study were recruited from the Department of Gynecologic Endocrinology and Reproductive
Medicine of Innsbruck Medical University. We approached reproductive-age women, $18-45$ years of age, who were being evaluated at our clinic for irregular menses, hirsutism, and/or infertility between February and December 2009.

We classified the subjects based on the diagnosis of PCOS according to the Rotterdam Consensus criteria [5]. Biochemical hyperandrogenemia was defined as total testosterone concentration $\geq 0.4 \mathrm{ng} / \mathrm{ml}$, as validated in our laboratory. We made further subgroupings based on the presence of IR defined as a HOMA Index of $\geq 2.4$ [23]. In selected cases, the presence of IR was further confirmed by a modified three-hour glucose tolerance test with an AUCinsulin of $>12,000 \mu \mathrm{IU} * 180 \mathrm{~min} / \mathrm{mL}$ defined as IR. Metabolic syndrome was defined according to the NCEP-ATP-III guidelines as the presence of at least three of five criteria: fasting glucose $\geq 110 \mathrm{mg} / \mathrm{dL}$, waist $>88 \mathrm{~cm}$, blood pressure $\geq 130 / \geq 85$, triglycerides $\geq 150$ $\mathrm{mg} / \mathrm{dL}$, and HDL $\leq 50 \mathrm{mg} / \mathrm{dL}$ [24]. The control group consisted of women in whom PCOS and/or non-classical adrenal hyperplasia (NCAH) were excluded. Most of the patients in the control group were diagnosed with either male factor infertility or tubal infertility.

Exclusion criteria included a history of endometriosis, diabetes, thyroid dysfunction or known metabolic disorders, chronic inflammatory conditions, including rheumatologic conditions. None of the women included in this study were taking oral contraceptives, hormonal medications, or insulin-sensitizing drugs. The study was approved by the Ethics Committee of Innsbruck Medical University. All participating subjects provided written informed consent.

\section{Clinical and laboratory data collection}

Clinical evaluation of the subjects included menstrual history and the presence of acne, hirsutism (evaluated with the Ferriman-Gallwey score) [25], seborrhoea, and androgenic alopecia. BMI, waist circumference, hip circumference, systolic and diastolic blood pressure as well as the appearance of the ovaries on ultrasound were recorded. Subjects underwent a peripheral blood draw after overnight fasting on cycle day 3-5 following a spontaneous or progesterone-induced bleed to determine a metabolic (total cholesterol, LDL cholesterol, HDL cholesterol, triglycerides, fasting glucose, fasting insulin) and hormone panel (luteinizing hormone (LH), follicle-stimulating hormone (FSH), estradiol (E2), dehydroepiandrosterone sulfate (DHEAS), testosterone, sex hormone-binding globulin (SHBG), free androgen index (FAI), prolactin and thyroidstimulating hormone (TSH)). A separate blood sample collected for measurement of afamin was centrifuged at low speed, aliquoted and the serum stored at $-80^{\circ} \mathrm{C}$ until analysis.

In addition, women with suspected PCOS underwent a $3 \mathrm{~h}, 75 \mathrm{~g}$ load oral glucose tolerance test (OGTT) for 
evaluation of the presence and degree of IR, as previously described [9]. In all women meeting criteria for PCOS late onset and heterozygous non-congenital adrenal hyperplasia were excluded with an ACTH stimulation test following overnight dexamethasone suppression, as previously described by Lejeune-Lenain [26].

\section{Laboratory analyses}

Hormone concentrations were measured by specific commercially available immunoassays. Total testosterone was determined using a radioimmunoassay (RIA) method with a Coat-a-count In-vitro Diagnostic Test Kit (Diagnostic Products Corporation, Los Angeles, CA, USA) in a Siemens Immulite 2000 Immunoassay system. The sensitivity of this assay was $0.04 \mu \mathrm{g} / \mathrm{L}$ and the intraassay and inter-assay coefficient of variation $(\mathrm{CV})$ for the range of values pertinent to our patients was less than $10 \%$. The free androgen index (FAI) was calculated as follows: total testosterone $(\mathrm{nmol} / \mathrm{L}) \times 100 / \mathrm{SHBG}(\mathrm{nmol} / \mathrm{L})$.

Afamin serum concentrations were quantified in duplicate by custom-made sandwich-type ELISA using specific mono- and polyclonal antibodies recognizing human afamin (MicroCoat Biotechnologie, Bernried, Germany), as previously described [21,22,27]. Within-run and total CV were $3.3 \%$ and $6.2 \%$, respectively, at a mean concentration of $73 \mathrm{mg} / \mathrm{L}$ [27].

The lipid profile including cholesterol and triglycerides as well as fasting glucose and insulin were determined using routine automated laboratory methods. Glucose was measured with the GOD-PAP method (glucose oxidase) and Roche/Hitachi 917 with an intra-assay CV of $0.9 \%$ and an inter-assay CV of $1.9 \%$. Insulin was determined by electrochemiluminescence immunoassay (ECLIA) with an intra-assay CV of $1.9 \%$ and an inter-assay CV of $2.6 \%$.

\section{Statistical analyses}

Baseline characteristics of the two groups were compared using $\mathrm{T}$ test, chi-square, and Fisher's exact test, where appropriate. Median concentrations of afamin were compared between groups using non-parametric tests, the Mann-Whitney test for two-group comparisons, and the Kruskal-Wallis test for multi-group comparisons. Bivariate relations between afamin and covariates were analyzed with Spearman's Rank Correlation Coefficient, and linear regression was used to test which variables were independent predictors of afamin level. The pre-selected independent variables evaluated were BMI, fasting insulin, HOMA Index, triglycerides, and LDL cholesterol. Statistical analyses were performed with GraphPad Prism, Version 6.01 (GraphPad Software, San Diego, CA, USA), and SPSS 22 (SPSS, Inc., Chicago, IL, USA).

G*Power 3.1.2 (Kiel, Germany) was used to calculate the sample size needed for this study. Using our previously published normal population median of serum afamin of $64 \mathrm{mg} / \mathrm{L}$ [27] and setting an $\alpha$ error probability of 0.05 and power (1- $\beta$ error probability) of 0.80 , we performed a two-tailed sample size calculation to detect a difference of $12 \%$ between non-PCOS and PCOS subjects. A minimum of 90 subjects was needed to achieve adequate power to detect this difference.

\section{Results}

We recruited 102 women for this study, 53 with PCOS and 49 without PCOS. Baseline characteristics, hormone concentrations and metabolic profiles of the study subjects are presented in Table 1. Women with PCOS were younger, had a higher BMI and waist circumference, and were more likely to have cycle irregularities, seborrhoea, and hirsutism. As expected, women with PCOS had higher concentrations of $\mathrm{LH}$ and testosterone, a higher

Table 1 Anthropometric characteristics, hormone concentrations and metabolic profiles of subjects

\begin{tabular}{|c|c|c|c|}
\hline Parameter & $\begin{array}{c}\text { PCOS } \\
(n=53) \\
\text { mean } \pm \text { SD or } \\
{ }^{*} \text { median } \pm \text { IQR. }\end{array}$ & $\begin{array}{c}\text { Controls } \\
(n=49) \\
\text { mean } \pm \text { SD or } \\
{ }^{*} \text { median } \pm \text { IQR. }\end{array}$ & $p$ \\
\hline Age yrs & $27.70 \pm 5.33$ & $32.14 \pm 7.39$ & 0.001 \\
\hline$B M I \mathrm{~kg} / \mathrm{m}^{2}$ & $28.37 \pm 7.14$ & $23.278 \pm 4.34$ & 0.001 \\
\hline Waist circumference cm & $99.33 \pm 13.22$ & $96.00 \pm 9.90$ & 0.012 \\
\hline Blood pressure systolic $\mathrm{mmHg}$ & $124.39 \pm 12.78$ & $122.55 \pm 12.76$ & 0.532 \\
\hline Blood pressure diastolic $\mathrm{mmHg}$ & $83.80 \pm 12.22$ & $79.74 \pm 9.97$ & 0.125 \\
\hline$L H U / L^{*}$ & $8.00 \pm 5.70$ & $4.35 \pm 2.98$ & 0.001 \\
\hline FSH U/L* & $5.85 \pm 1.63$ & $6.85 \pm 3.25$ & 0.037 \\
\hline$L H / F S H^{*}$ & $1.51 \pm 1.30$ & $0.61 \pm 0.37$ & 0.001 \\
\hline$E 2 n g / L^{*}$ & $49.50 \pm 22.25$ & $39.00 \pm 28.50$ & 0.060 \\
\hline DHEAS $m g / L^{*}$ & $1.94 \pm 1.70$ & $1.34 \pm 0.95$ & 0.008 \\
\hline Testosterone $\mu \mathrm{g} / \mathrm{L}^{*}$ & $0.54 \pm 0.36$ & $0.22 \pm 0.14$ & 0.001 \\
\hline SHBG nmol/ $\mathrm{L}^{*}$ & $35.45 \pm 38.18$ & $55.45 \pm 35.65$ & 0.003 \\
\hline FAl Index* & $5.85 \pm 6.14$ & $1.78 \pm 1.61$ & 0.001 \\
\hline Fasting glucose $\mathrm{mg} / \mathrm{dL}$ & $87.30 \pm 6.94$ & $87.83 \pm 8.68$ & 0.734 \\
\hline Fasting insulin $U / L^{*}$ & $9,8 \pm 10,50$ & $6,65 \pm 5,92$ & 0.011 \\
\hline Total cholesterol mg/dL & $189.09 \pm 33.10$ & $181.43 \pm 32.97$ & 0.267 \\
\hline HDL cholesterol mg/dL & $57.21 \pm 16.79$ & $60.87 \pm 15.21$ & 0.274 \\
\hline LDL cholesterol mg/dL & $108.00 \pm 27.5$ & $96.50 \pm 36.25$ & 0.089 \\
\hline Triglycerides $m g / d L^{*}$ & $101.00 \pm 61.00$ & $81.50 \pm 44.75$ & 0.031 \\
\hline AUC Insulin $\mu U^{*} 180 \mathrm{~min} / \mathrm{mL}^{*}$ & $9935 \pm 9762$ & & \\
\hline AUC Glucose $\mathrm{mg} / \mathrm{dl}^{*} 180 \mathrm{~min} / \mathrm{mL}$ & $18606 \pm 2762$ & $16510 \pm 1136$ & 0.201 \\
\hline HOMA Index* & $2.16 \pm 2.37$ & $1.39 \pm 1.24$ & 0.013 \\
\hline Afamin $m g / L^{*}$ & $66.43 \pm 19.60$ & $62.33 \pm 18.53$ & 0.745 \\
\hline
\end{tabular}

Values of variables are given as mean \pm standard deviation (SD), or *median \pm IQR. Statistically significant $p$ values are shown as bold numbers. AUC: area under the curve. 
FAI and LH/FSH ratio and lower SHBG concentrations than did non-PCOS women.

Women with PCOS had significantly higher mean fasting insulin and triglyceride concentrations and a higher mean HOMA Index and were more likely to have IR ( $45.3 \%$ vs. $21.4 \%, p=0.015)$, but not more likely to meet the criteria for MetS $(20.75 \%$ vs. $8.11 \%, \mathrm{p}=0.103)$ than were those without PCOS.

Median afamin concentration was $66.43+/-19.60 \mathrm{mg} / \mathrm{L}$ in the PCOS and $62.33+/-18.53 \mathrm{mg} / \mathrm{L}$ in the non-PCOS group, a statistically non-significant difference $(\mathrm{p}=0.745$, Table 1). Further separation of the subjects according to the presence of IR revealed a significant difference in afamin concentrations between the four groups $(\mathrm{H}(3)=$ 13.415, $\mathrm{p}=0.004$; Figure 1). Using Dunn's multiple comparison test, we found significantly higher median afamin levels in PCOS with IR as compared to PCOS without IR and controls without IR, namely: PCOS with IR: $73.06+/-27.36 \mathrm{mg} / \mathrm{L}$, PCOS without IR: $64.25+/-17.41$ $\mathrm{mg} / \mathrm{L}$, controls without IR: $60.44+/-21.03 \mathrm{mg} / \mathrm{L}, \mathrm{p}=0.033$ and $\mathrm{p}=0.011$. However, no difference in afamin levels was seen when comparing controls with IR and controls without IR $(76.20+/-27.96 \mathrm{mg} / \mathrm{L}$ and $60.44+/-21.03 \mathrm{mg} / \mathrm{L}$ $\mathrm{p}=0.235)$.

We further conducted a one-way analysis of covariance (ANCOVA) using afamin as the dependent variable, PCOS as the independent variable (two levels) and fasting insulin as the covariate. ANCOVA (between-subjects factor: PCOS, control) showed no main effects of PCOS $F(1,92)=0.115, \mathrm{p}=0.736)$, but revealed that it is

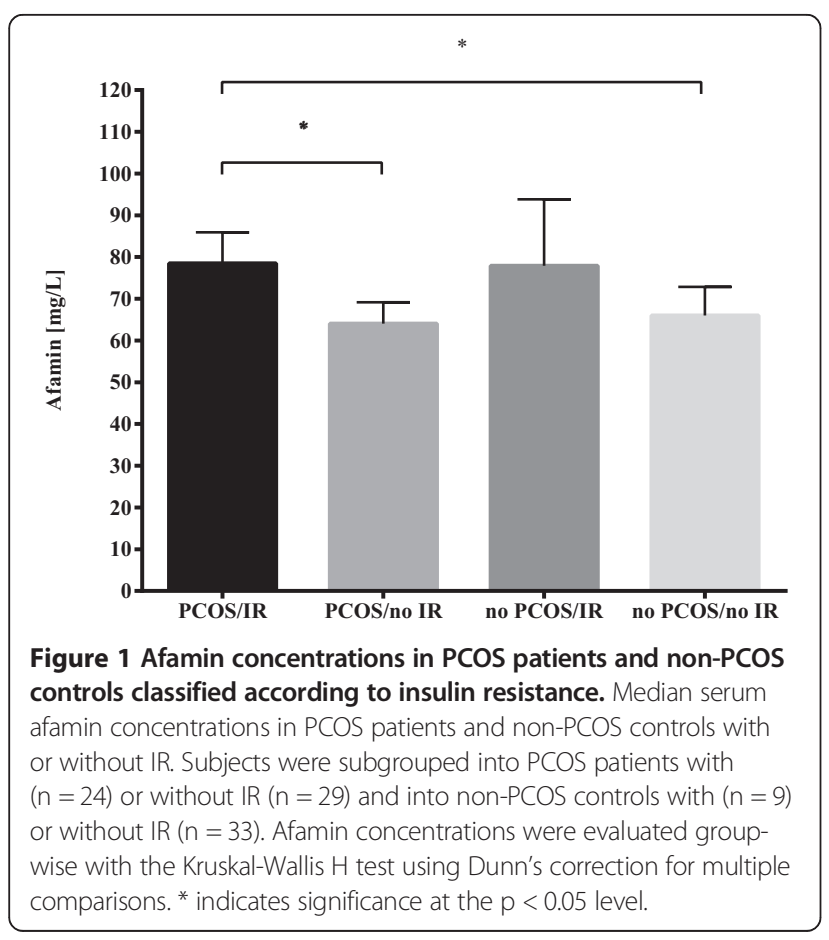

the insulin concentration that mainly determines afamin value $F(1.92)=10.06, \mathrm{p}=0.002, \eta \mathrm{p}^{2}=0.10$. When controlling for fasting insulin, the adjusted means for afamin were $70.42 \mathrm{mg} / \mathrm{L}(\mathrm{SE}=2.77)$ and $69.14 \mathrm{mg} / \mathrm{L}(\mathrm{SE}=2.45)$ for the PCOS and the control group, respectively.

On individual correlations using the Spearman coefficient $r$, the following parameters correlated significantly with afamin concentrations: BMI $(r=0.231, p=0.02)$, waist/hip ratio $(\mathrm{r}=0.310, \mathrm{p}=0.019)$, fasting insulin $(\mathrm{r}=$ 0.313, $\mathrm{p}=0.002)$, LDL cholesterol $(\mathrm{r}=0.257, \mathrm{p}=0.013)$, HOMA Index $(\mathrm{r}=0.310, \mathrm{p}=0.002)$, and triglycerides $(\mathrm{r}=$ $0.372, \mathrm{p}=0.001$ ) as the only criterion of metabolic syndrome. No statistically significant correlation was seen between afamin and other parameters of metabolic syndrome, including waist circumference $(\mathrm{r}=0.259, \mathrm{p}=$ $0.052)$, systolic or diastolic blood pressure $(r=0.194$, $\mathrm{p}=0.085 ; \mathrm{r}=0.199, \mathrm{p}=0.077)$, fasting glucose $(\mathrm{r}=0.055$, $\mathrm{p}=0.582)$ or HDL $(\mathrm{r}=-0.166, \mathrm{p}=0.111)$.

Afamin correlated with FAI $(\mathrm{r}=387, \mathrm{p}=0.0001)$ but not with testosterone $(r=0.015, p=0.879)$, SHBG $(r=-0.134$, $\mathrm{p}=0.182)$, FSH $(\mathrm{r}=-0.156, \mathrm{p}=0.120)$ or $\mathrm{E} 2(\mathrm{r}=-0.147$, $\mathrm{p}=0.145)$.

However, on multivariable linear regression including as independent variables selected parameters that significantly correlated with afamin (BMI, LDL cholesterol, triglycerides, HOMA Index and fasting insulin) only triglyceride concentration was seen to be an independent predictor of afamin concentration ( $\beta$ coefficient $=0.271$, $\mathrm{p}=0.019$, Durbin-Watson 1.468).

Finally, we compared median afamin concentrations between those with and without diagnosed metabolic syndrome, independent of the presence of PCOS. We found markedly elevated afamin concentrations in women with metabolic syndrome versus without, values that were more than $12 \mathrm{mg} / \mathrm{L}$ higher than in women without metabolic syndrome, as shown in Figure 2 $(77.43+/-28.60 \mathrm{mg} / \mathrm{L}$ and $65.08+/-18.03 \mathrm{mg} / \mathrm{L}$, respectively, $(\mathrm{p}=0.010))$.

\section{Discussion}

Women with PCOS have a higher prevalence of several risk factors for CVD, including dyslipidemia, IR, type 2 diabetes and hypertension, than do age-matched, nonPCOS women [28]. Although these risk factors are exacerbated by the concomitant obesity seen in some PCOS women, they are also present in lean, non-obese women with PCOS, many of whom exhibit IR $[9,29,30]$. Due to the elevated lifetime risk of developing metabolic syndrome and its subsequent atherosclerosis and CVD complications, the Androgen Excess and Polycystic Ovary Syndrome (AE-PCOS) Society has published specific recommendations for screening and management of women with PCOS [31]. Early identification of women at risk has clinical implications, because lifestyle 


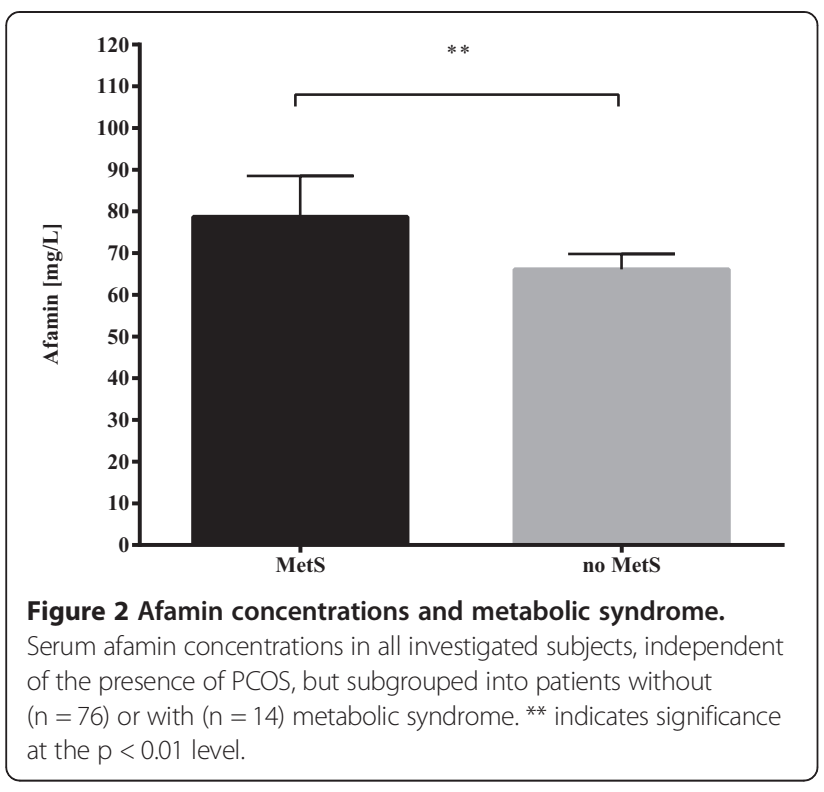

modifications or medications such as insulin-sensitizing and/or cholesterol-lowering drugs may be initiated for primary CVD prevention.

The present study found no difference in serum concentrations of afamin, a vitamin E-binding protein, between women with PCOS and non-PCOS controls. When sub-grouping all investigated subjects according to the presence of IR, however, women with IR exhibited higher afamin concentrations, regardless of the presence of PCOS. A one-way analysis of covariance showed that it is fasting insulin and not the diagnosis of PCOS that correlates with afamin. Afamin was markedly higher in women already diagnosed with metabolic syndrome. These findings corroborate those of a previous population-based study that likewise showed that afamin concentrations were associated with the number of metabolic syndrome criteria in groups of men and women with mean age between 50 and 60 [19]. Despite evaluating a much younger cohort of women with an average age of around 30, the present study nonetheless found afamin levels to be similarly elevated. Age has, however, no or very little influence on afamin concentrations, as shown in a group of blood donors with different age subgroups [27]. Thus, our findings confirm that young women with PCOS are more likely to have metabolic syndrome, and afamin might serve as a discriminatory predictive parameter of IR in young PCOS patients. As such, PCOS can be considered a confounder in the relationship between afamin and IR.

This study confirms and extends a recently published independent investigation of afamin in PCOS patients [32]. The two studies differ, however, in study design and investigated key parameters. While Köninger et al. analysed retrospective data in a cross-sectional study, our patients were prospectively recruited. Furthermore, we were able to analyse the prevalence of IR based on HOMA Index as well as the prevalence of metabolic syndrome in both the PCOS- and the non-PCOS groups and provide information on the diagnosis of metabolic syndrome. In this way it was shown that, similar to the general population [19], afamin concentrations are associated with the prevalence of metabolic syndrome in PCOS patients. The present study did not find women with PCOS to have higher afamin levels than controls, as reported by Köninger et al. The differing results might be partially explained by the lack of prevalence data on IR and metabolic syndrome for the control group in the Köninger study, which could greatly influence the results if the control group consisted of a population almost exclusively having normal insulin sensitivity. Studies including larger numbers of subjects with documented IR levels and metabolic syndrome will therefore be necessary in future for final confirmation.

Of the laboratory parameters for metabolic syndrome evaluated, triglyceride level was the sole independent predictor of afamin concentrations. Interestingly, Dokras et al. reported that an elevated serum triglyceride/HDL cholesterol ratio was highly sensitive and specific for the detection of metabolic syndrome in women with PCOS [33]. The study by Kronenberg et al. [19] likewise found that afamin most strongly correlated with triglycerides and waist circumference in older populations of women and men.

Kronenberg et al. followed their subjects prospectively for five years and were able to show that each incremental increase in afamin of $10 \mathrm{mg} / \mathrm{L}$ increased the probability of being diagnosed with metabolic syndrome by $74 \%$. Unfortunately, at this time we have no longitudinal data available for the women in the present cohort, but we are likewise following up the women in this study and re-evaluating afamin concentrations and components of metabolic syndrome in the hope that afamin may be useful as a predictive parameter for the development of metabolic syndrome in younger women with insulin resistance.

\section{Conclusions}

It is only in recent years that more attention has been paid to the metabolic abnormalities of PCOS, and not just the syndrome's implications for infertility and infertility treatment. As discussed in a recent review by Bates and Legro [34], understanding the true health implications of PCOS is difficult due to the heterogeneous nature of the condition and the lack of long-term cohort studies. Thus, large prospective longitudinal studies, ideally involving many ethnic groups, are needed to improve our understanding of the long-term health risks associated with PCOS. The results of the study at hand show that elevated afamin concentrations are associated 
with the presence of metabolic syndrome in young women and may potentially serve as an independent predictor for the development of metabolic syndrome in at-risk women, especially those with insulin resistance. For this purpose, however, prospective studies with sufficiently large cohorts of well-characterised PCOS women will have to be performed.

\section{Competing interests}

$\mathrm{HD}$ is owner and shareholder of Vitateq Biotechnology GmbH, Innsbruck, Austria, a spin-off biotech company from Innsbruck Medical University, holding several patents related to research described in this article. All other coauthors report no conflict of interest.

\section{Authors' contributions}

BS was responsible for study design, statistical analyses, data interpretation, and manuscript drafting. EM was responsible for data collection, statistical analysis and manuscript drafting. FL contributed to statistical analysis, data interpretation and manuscript drafting. LW contributed to study design, data interpretation and manuscript drafting. HD conceived the study, participated in its design and coordination and helped draft and revise the manuscript. All authors read and approved the final manuscript.

\section{Acknowledgements}

We thank Linda Fineder for expert technical assistance and Mary Heaney Margreiter, interpreter/editor, for native-speaker language editing. This work was supported by grants to H.D. from the Austrian Research Fund (P19969-B11) and the Austrian National Bank (12529).

\section{Author details}

'Department of Gynecologic Endocrinology and Reproductive Medicine, Innsbruck Medical University, Anichstrasse 35, A-6020 Innsbruck, Austria. ${ }^{2}$ Department of Medical Genetics, Division of Genetic Epidemiology, Molecular and Clinical Pharmacology, Innsbruck Medical University, Schöpfstrasse 41, A-6020 Innsbruck, Austria. ${ }^{3}$ Vitateq Biotechnology GmbH, Innsbruck, Austria.

Received: 31 May 2014 Accepted: 9 August 2014

Published: 10 September 2014

\section{References}

1. Stein IF, Leventhal ML: Amenorrhoea associated with bilateral polycystic ovaries. Am J Obstet Gynecol 1935, 29:181-191.

2. Fauser BC, Tarlatzis BC, Rebar RW, Legro RS, Balen AH, Lobo R, Carmina E, Chang J, Yildiz BO, Laven JS, Boivin J, Petraglia F, Wijeyeratne CN, Norman RJ, Dunaif A, Franks S, Wild RA, Dumesic D, Barnhart K: Consensus on women's health aspects of polycystic ovary syndrome (PCOS): the Amsterdam ESHRE/ASRM-Sponsored 3rd PCOS Consensus Workshop Group. Fertil Steril 2012, 97:28-38. e25.

3. Sirmans SM, Pate KA: Epidemiology, diagnosis, and management of polycystic ovary syndrome. Clin Epidemiol 2013, 6:1-13.

4. Goodarzi MO, Dumesic DA, Chazenbalk G, Azziz R: Polycystic ovary syndrome: etiology, pathogenesis and diagnosis. Nat Rev Endocrinol 2011, 7:219-231.

5. Rotterdam EA-SPcwg;: Revised 2003 consensus on diagnostic criteria and long-term health risks related to polycystic ovary syndrome (PCOS). Hum Reprod 2004, 19:41-47.

6. Dunaif A, Segal KR, Futterweit W, Dobrjansky A: Profound peripheral insulin resistance, independent of obesity, in polycystic ovary syndrome. Diabetes 1989, 38:1165-1174.

7. Apridonidze T, Essah PA, luorno MJ, Nestler JE: Prevalence and characteristics of the metabolic syndrome in women with polycystic ovary syndrome. J Clin Endocrinol Metab 2005, 90:1929-1935.

8. Talbott EO, Zborowski JV, Rager JR, Boudreaux MY, Edmundowicz DA, Guzick DS: Evidence for an association between metabolic cardiovascular syndrome and coronary and aortic calcification among women with polycystic ovary syndrome. J Clin Endocrinol Metab 2004, 89:5454-5461.
9. Lunger F, Wildt $L$, Seeber B: Accurate screening for insulin resistance in PCOS women using fasting insulin concentrations. Gynecol Endocrinol 2013, 29:541-544.

10. Eckel RH, Alberti KG, Grundy SM, Zimmet PZ: The metabolic syndrome. Lancet 2010, 375:181-183.

11. Alberti KG, Eckel RH, Grundy SM, Zimmet PZ, Cleeman Jl, Donato KA Fruchart JC, James WP, Loria CM, Smith SC Jr, International Diabetes Federation Task Force on Epidemiology and Prevention; Hational Heart, Lung, and Blood Institute; American Heart Association; World Heart Federation; International Atherosclerosis Society; International Association for the Study of Obesity: Harmonizing the metabolic syndrome: a joint interim statement of the International Diabetes Federation Task Force on Epidemiology and Prevention; National Heart, Lung, and Blood Institute; American Heart Association; World Heart Federation; International Atherosclerosis Society; and International Association for the Study of Obesity. Circulation 2009, 120:1640-1645.

12. Ford ES, Giles WH, Mokdad AH: Increasing prevalence of the metabolic syndrome among u.s. Adults. Diabetes Care 2004, 27:2444-2449.

13. Third Report of the National Cholesterol Education Program (NCEP): Expert panel on detection, evaluation, and treatment of high blood cholesterol in adults (adult treatment panel III) final report. Circulation 2002, 106:3143-3421.

14. Ford ES: Risks for all-cause mortality, cardiovascular disease, and diabetes associated with the metabolic syndrome: a summary of the evidence. Diabetes Care 2005, 28:1769-1778.

15. Galassi A, Reynolds K, He J: Metabolic syndrome and risk of cardiovascular disease: a meta-analysis. Am J Med 2006, 119:812-819.

16. Gami AS, Witt BJ, Howard DE, Erwin PJ, Gami LA, Somers VK, Montori VM: Metabolic syndrome and risk of incident cardiovascular events and death: a systematic review and meta-analysis of longitudinal studies. J Am Coll Cardiol 2007, 49:403-414.

17. Haffner SM, Lehto S, Ronnemaa T, Pyorala K, Laakso M: Mortality from coronary heart disease in subjects with type 2 diabetes and in nondiabetic subjects with and without prior myocardial infarction. N Engl J Med 1998, 339:229-234.

18. Legro RS: Polycystic ovary syndrome and cardiovascular disease: a premature association? Endocr Rev 2003, 24:302-312.

19. Kronenberg F, Kollerits B, Kiechl S, Lamina C, Kedenko L, Meisinger C, Willeit J, Huth C, Wietzorrek G, Altmann ME, Thorand B, Melmer A, Dähnhardt D, Santer P, Rathmann W, Paulweber B, Koenig W, Peters A, Adham IM, Dieplinger $\mathrm{H}$ : Plasma concentrations of afamin are associated with the prevalence and development of metabolic syndrome. Circ Cardiovasc Genet 2014, doi:10.1161/CIRCGENETICS.113.000654, in press.

20. Lichenstein HS, Lyons DE, Wurfel MM, Johnson DA, MCGinley MD, Leidli JC, Trollinger DB, Mayer JP, Wright SD, Zukowski MM: Afamin is a new member of the albumin, a-fetoprotein, and vitamin D-binding protein gene family. J Biol Chem 1994, 269:18149-18154.

21. Jerkovic L, Voegele AF, Chwatal S, Kronenberg F, Radcliffe CM, Wormald MR, Lobentanz EM, Ezeh B, Eller P, Dejori N, Dieplinger B, Lottspeich F, Sattler W, Uhr M, Mechtler K, Dwek RA, Rudd PM, Baier G, Dieplinger H: Afamin is a novel human vitamin E-binding glycoprotein characterization and in vitro expression. J Proteome Res 2005, 4:889-899.

22. Voegele AF, Jerkovic L, Wellenzohn B, Eller P, Kronenberg F, Liedl KR, Dieplinger $\mathrm{H}$ : Characterization of the vitamin E-binding properties of human plasma afamin. Biochemistry 2002, 41:14532-14538.

23. Radikova Z, Koska J, Huckova M, Ksinantova L, Imrich R, Vigas M, Trnovec T, Langer P, Sebokova E, Klimes I: Insulin sensitivity indices: a proposal of cut-off points for simple identification of insulin-resistant subjects. Exp Clin Endocrinol Diabetes 2006, 114:249-256.

24. Grundy SM, Cleeman JI, Daniels SR, Donato KA, Eckel RH, Franklin BA, Gordon DJ, Krauss RM, Savage PJ, Smith SC Jr, Spertus JA, Costa F, American Heart Association, National Heart, Lung, and Blood Institute: Diagnosis and management of the metabolic syndrome: an American Heart Association/National Heart, Lung, and Blood Institute Scientific Statement. Circulation 2005, 112:2735-2752.

25. Ferriman D, Gallwey JD: Clinical assessment of body hair growth in women. J Clin Endocrinol Metab 1961, 21:1440-1447.

26. Lejeune-Lenain C, Cantraine F, Dufrasnes M, Prevot F, Wolter R, Franckson $J R$ : An improved method for the detection of heterozygosity of congenital virilizing adrenal hyperplasia. Clin Endocrinol (Oxf) 1980, 12:525-535. 
27. Dieplinger B, Egger M, Gabriel C, Poelz W, Morandell E, Seeber B, Kronenberg F, Haltmayer M, Mueller T, Dieplinger H: Analytical characterization and clinical evaluation of an enzyme-linked immunosorbent assay for measurement of afamin in human plasma. Clin Chim Acta 2013, 425:236-241.

28. Legro RS: Type 2 diabetes and polycystic ovary syndrome. Fertil Steril 2006, 86(Suppl 1):S16-S17.

29. Diamanti-Kandarakis E, Kandarakis H, Legro RS: The role of genes and environment in the etiology of PCOS. Endocrine 2006, 30:19-26.

30. Nestler JE, Jakubowicz DJ: Lean women with polycystic ovary syndrome respond to insulin reduction with decreases in ovarian P450c17 alpha activity and serum androgens. J Clin Endocrinol Metab 1997, 82:4075-4079.

31. Wild RA, Carmina E, Diamanti-Kandarakis E, Dokras A, Escobar-Morreale HF, Futterweit W, Lobo R, Norman RJ, Talbott E, Dumesic DA: Assessment of cardiovascular risk and prevention of cardiovascular disease in women with the polycystic ovary syndrome: a consensus statement by the Androgen Excess and Polycystic Ovary Syndrome (AE-PCOS) Society. J Clin Endocrinol Metab 2010, 95:2038-2049.

32. Koninger A, Edimiris P, Koch L, Enekwe A, Lamina C, Kasimir-Bauer S, Kimmig $\mathrm{R}$, Dieplinger $\mathrm{H}$ : Serum concentrations of afamin are elevated in patients with polycystic ovary syndrome. Endocr Connect 2014, 3:120-126.

33. Dokras A, Bochner M, Hollinrake E, Markham S, Vanvoorhis B, Jagasia DH: Screening women with polycystic ovary syndrome for metabolic syndrome. Obstet Gynecol 2005, 106:131-137.

34. Bates GW, Legro RS: Longterm management of Polycystic Ovarian Syndrome (PCOS). Mol Cell Endocrinol 2013, 373:91-97.

doi:10.1186/1477-7827-12-88

Cite this article as: Seeber et al:: Afamin serum concentrations are associated with insulin resistance and metabolic syndrome in polycystic ovary syndrome. Reproductive Biology and Endocrinology 2014 12:88.

\section{Submit your next manuscript to BioMed Central and take full advantage of:}

- Convenient online submission

- Thorough peer review

- No space constraints or color figure charges

- Immediate publication on acceptance

- Inclusion in PubMed, CAS, Scopus and Google Scholar

- Research which is freely available for redistribution 